\title{
UN CAS D'HYPERTHERMIE MALIGNE SURVENU EN CLINIQUE DENTAIRE
}

\author{
André Martel, Linda Spigelblatt, Luc Perreault, et Robert Rosenfeld
}

\section{Histoire de cas}

UNE FILletTe de 5.5 ans, J.T., pesant $15.5 \mathrm{~kg}$, est admise à une clinique dentaire pour un travail de restauration ( 8 dents). L'anamnèse de l'enfant est négatif et le questionnaire ne révèle aucune particularité, sauf une légère toux chronique. L'examen physique est normal à l'exception d'une hypertrophie modérée des amygdales. L’enfant n’a pas reçu de prémédication (avant l’induction). Le rythme cardiaque est régulier à $100 \cdot \mathrm{min}$ et la tension artérielle systolique est de $90 \mathrm{~mm} \mathrm{Hg}$ (11.97 kPa).

Après l'installation d'un soluté dextrosé à 5 pour cent. l'anesthésie est induite avec une dose endoveneuse de $25 \mathrm{mg}$ de Méthohexital Sodique, suivie d'administration de $0.1 \mathrm{mg}$ d'atropine et de $20 \mathrm{mg}$ de Succinylcholine. L'enfant est intubée par voie nasale et l'anesthésie maintenue à l'aide d'un mélange de $3 \mathrm{~L} \cdot$ min d'oxygène, de $4 \mathrm{~L} \cdot \min$ de protoxyde d'azote, et d'halothane, administré par un circuit pédiatrique Ayre T. La ventilation est assistée manuellement, le débit du soluté ajusté à $60 \mathrm{ml} \cdot h e u r e$ et les signes vitaux monitorés par un stéthoscope précordial et un moniteur cardiaque avec une dérivation V-2.

Dès l'induction, l'anesthésiste note une rigidité modérée du maxillaire inférieur mais l'intubation est facile. La température est alors monitorée en permanence par voie rectale. Deux minutes après l'intubation, la température rectale est de $37.7^{\circ} \mathrm{C}$, ce qui nous semble acceptable vu I'histoire de toux et amygdalite. Le pouls est de 130 minute avec un épisode transitoire d'extra-systoles ventriculaires isolées.

Quarante-cinq minutes après l̈induction, la température rectale passe brusquement de $37.7^{\circ}$

André Martel, M.D., F.R.C.P.(C). Anesthésiste au Centre Dentaire Anjou Inc. et Hôpital B.M.P. Cowansville.

Linda Spigelblatt. M.D.. F.R.C.P.(C) and Robert Rosenfeld, M.D., Department de Pédiatrie - Hôpital Maisonneuve-Rosemont.

Luc Perreault, M.D., Département d'AnesthésieRéanimation - Hôpital Maisonneuve-Rosemont.

Adresse postale: Luc Perreault, M.D., Hôpital Maisonneuve-Rosemont, Département d’AnesthésieRéanimation, 5415 blvd. l'Assomption. Montréal P.Q. Canada, H IT $2 \mathrm{M} 4$.
$\mathrm{C}$ à $38.0^{\circ} \mathrm{C}$ et à $38.8^{\circ} \mathrm{C}$ en cinq minutes. Le pouls s'accélère à $160 \mathrm{~min}$ et une légère cyanose est notée. Le diagnostic d'hyperthermie maligne est alors posé. Le dentiste termine rapidement la chirurgie pendant que le traitement suivant est entrepris : hyperventilation à 100 pour cent d'oxygène, soluté glacé de lactate Ringer à 80 $\mathrm{ml} \cdot$ heure, procainamide $100 \mathrm{mg}$ endoveineuse en 20 minutes, suivis d'une perfusion à 100 $\mathrm{mg}$-heure, $25 \mathrm{mmol}$ de $\mathrm{NaHCO}_{3}$ endoveineuse, insertion d'une sonde nasogastrique et lavage d'estomac à l'eau glacée, immersion de l'enfant dans des sacs de glace concassée. Une ambulance est demandée et l'hôpital est averti de l'arrivée de l'enfant.

La température passe à $40.8^{\circ} \mathrm{C}$ mais redescend à $37.7^{\circ} \mathrm{C}$ lors du départ pour l'hôpital, 30 minutes plus tard. L'enfant reprend conscience mais est gardée intubée. Les parents sont avisés de son état.

La fillette arrive à l'hôpital environ une heure et demie après le début de l'anesthésie. Les manœuvres de refroidissement de surface sont continuées. Un prélèvement artériel avec une $\mathrm{FI}_{\mathrm{O}_{2}}$ de 0.21 en respiration spontanée nous donne les résultats suivants: $\mathrm{Pa}_{02} 12.83 \mathrm{kPa}$ (96.5 torr), $\mathrm{cH}^{+} 57.54 \mathrm{nmol} \cdot 1$ ( $\left.\mathrm{pH} 7.24\right) \mathrm{PCO}_{2} 5.35 \mathrm{kPa}(40.2$ torr), E.B. -9.5. L'enfant est alors extubée et on lui administre 25 mmol de $\mathrm{NaHCO}_{3}$ par voie endoveineuse. Après l'installation d'une sonde urinaire on lui donne $12.5 \mathrm{gm}$ de mannitol par voie endoveineuse. Un thermomètre rectal est introduit après un certain retard, et révèle que la température est de $31.9^{\circ} \mathrm{C}$. Le réchauffement se fait graduellement et la réanimation est complétée deux heures après le début de l'anesthésie.

L'enfant a évolué favorablement. Une analyse des gaz sanguins, 60 minutes après son arrivée, montre $\mathrm{cH}^{+} 40.74 \mathrm{n} \mathrm{mol} \cdot 1$ ( $\left.\mathrm{pH} 7.39\right), \mathrm{PCO}_{2} 5.59 \mathrm{kPa}$ (42 torr) des E.B. à 0 et une composante métabolique normale. Nous pouvons cesser la réfrigération et l'administration de procainamide. Les signes cliniques d'hyperthermie sont en nette régression, une heure après son arrivée à l'hôpital.

A son arrivée à l"hôpital, le dosage des CPK 


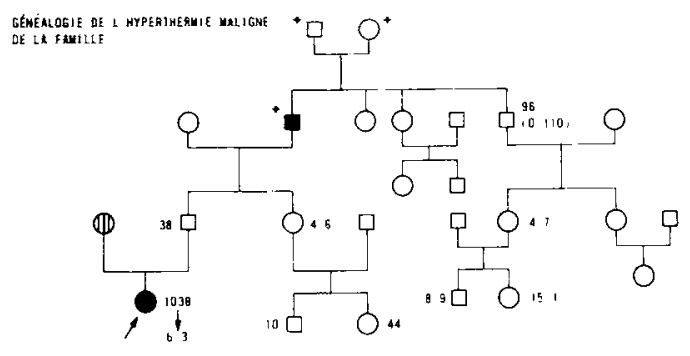

LEGEMOE

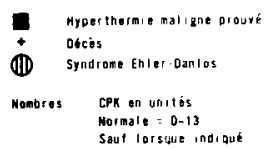

(normale 24-78 u), les électrolytes ainsi que était de 1038 u (normale 0-13 u), les LDH à $195 u$ l'azotémie dans les limites de la normale, la glycémie légèrement élevée à cause d'un soluté glucosé. Le dosage du calcium s'est avéré normal. L'analyse d'urine montraite une hémoglobinurie et myoglobinurie. Une radiographie pulmonaire revint normale. L'électrocardiogramme montra une tachycardie sinusale à 138minute avec des signes de surcharge ventriculaire droite. Trente-six (36) heures après son admission, la veille de Noël, l'enfant reçut son congé en excellente condition.

\section{Histoire familiale}

Cette fillette est la seule enfant de parents non-consanguins, Canadien-français. Vu l'épisode d'hyperthermie maligne survenu chez leur enfant, les parents sont de nouveau interviewés afin de connaître les complications anesthésiques qui auraient pu survenir dans la famille. Le père se rappelle que son père (grand-père paternel de l'enfant) est décédé à l'âge de 58 ans, quelques heures après une sympathectomie lombaire bilatérale sous anesthésie générale. La cause de la mort, vérifiée dans le dossier d'hospitalisation, a été déterminée comme étant une hyperthermie maligne. A l'époque aucun conseil n'a été donné à la famille. Cet incident n'a d'ailleurs pas été mentionné en clinique dentaire avant l'anesthésie de l'enfant. Le père de l'enfant dit avoir subi sans incident plusieurs interventions chirurgicales sous anesthésie. La tante paternelle et les cousins ont, eux aussi, subi sans complication des interventions chirurgicales sous anesthésie générale; aucun n'est porteur de syndrome musculaire suspect; la mère de l'enfant souffre d'un syndrome d'Ehler-Danlos et n'a connu aucune complication après plusieurs anesthésies générales.
Tel que déjà rapporté, il n'y a pas d'histoire familiale positive de myotonie congénitale ou de myopathie de Evans en association avec le syndrome d'hyperthermie maligne. ${ }^{1,2}$

Les résultats des CPK mesurés chez les parents et plusieurs membres de la famille apparaissent au tableau généalogique de l'hyperthermie dans cette lignée.

\section{Conseils genetiques}

La transmission de l'hyperthermie maligne comme caractère dominant avec pénétration variable a été expliquée aux parents et aux autres membres de la famille. ${ }^{3}$ L'enfant a été avisée de porter en permanence un bracelet Medic Alert et les parents ont été renseignés sur les agents anesthésiques qui doivent être évités. Le père est considéré comme porteur de la maladie jusqu'à preuve du contraire, même s'il a subi antérieurement des interventions chirurgicales sans complication anesthésique. ${ }^{2}$ Les membres de la famille considérés comme suspects à l'analyse des CPK seront soumis à une biopsie musculaire selon la technique décrite par Denborough. ${ }^{4}$

Les membres de la famille ont été avisés de renseigner leur médecin et leur dentiste de la présence d'hyperthermie maligne dans la famille.

\section{Discussion}

Notre patiente n'avait pas de strabisme, de ptose congénitale, d'hernie inguinale ou de cyphoscoliose, anomalies connues comme étant associées avec l'hyperthernie maligne. ${ }^{1.5} \mathrm{Ces}$ malformations se rencontrent spécialement chez les garçons et peuvent aussi s'accompagner de cryptorchidie.

L'enfant n'avait aucun signe visible du syndrome d'Ehler-Danlos, pathologie transmise comme caractère dominant avec pénétration variable et caractérisée par une hyperextensibilité des articulations et une hyperélasticité et fragilité de la peau. ${ }^{6}$

L'absence de l'élévation des CPK ne peut faire conclure avec certitude à l'absence de l'hyperthermie maligne chez un individu. ${ }^{7}$ Les CPK ont été refaits chez la fillette et ont donné des valeurs dans la limite inférieure de la normale. Ceci s'explique probablement par une déplétion temporaire du réservoir des enzymes à la suite de l'épisode d'hyperthermie. ${ }^{8}$

Le traitement énergique peut abaisser la température à des niveaux potentiellement dangereux, surtout chez les enfants, en raison de 
la grande surface corporelle. Nous croyons que ce danger augmente lors de l'arrêt de l'application de glace, alors que les tissus cutanés se reperfusent et qu'il peut $y$ avoir absorption rapide de froid dans la circulation centrale. ${ }^{9}$ Il est probable que la température de $31.9^{\circ} \mathrm{C}$ notée peu après le début du réchauffement de l'enfant soit due à ce phénomène. Heureusement dans ce cas, la température est revenue à la normale très rapidement sans causer de problème cardiaque.

\section{Conclusion}

L'intérêt de cette présentation porte surtout sur ce cas d'hyperthermie maligne survenu en clinique dentaire, qui a pu recevoir sur place, le traitement adéquat pour contrer l'évolution de l'hyperthermie. Depuis quelques années. la Corporation Professionnelle des Médecins du Québec exige que les médecins qui administrent l'anesthésie générale dans des centres hors des hôpitaux se procurent un équipement aussi complet que dans les hôpitaux afin de prévenir les complications anesthésiques possibles. L'anesthésiste possédait donc le matériel adéquat pour faire la réfrigération de la patiente ainsi que les médicaments nécessaires. Ces exigences sont d'ailleurs contenues dans le Guide d'exercice de l'Anesthésie, publié par la Corporation Professionnelle des Médecins du Québec en 1975. ${ }^{10}$

\section{RÉSUMÉ}

Un cas d'hyperthermie maligne, complication grave de l'anesthésie, est survenu chez une fillette de $5 \frac{1}{2}$ ans subissant une chirurgie dentaire en clinique dentaire. Grâce aux exigences actuelles pour la pratique de l'anesthésie dans les cliniques dentaires du Québec, ce type de complication anesthésique a pu être bien traité.

\section{SUMMARY}

Malignant hyperthermia, a life-threatening complication of anaesthesia, occurred in a $5 \frac{1}{2}-$ year-old girl undergoing dental surgery in a dental clinic. During induction, after $20 \mathrm{mg}$ of succinylcholine, the anaesthetist noted a moderate rigidity of the mandible that did not interfere with the tracheal intubation. However, after $45 \mathrm{~min}$ utes, the temperature rose to $38.8^{\circ} \mathrm{C}$, pulse was rapid at 160 per minute and the child was slightly cyanosed. The diagnosis of malignant hyper- thermia was made (the temperature eventually reached $40.8^{\circ} \mathrm{C}$ ). The child was immediately treated with refrigeration, procainamide and sodium bicarbonate. She was then moved to a hospital centre where the treatment was continued and she recovered completely.

After extensive investigation, we found that the paternal grandfather of the child died at age 58. several hours after a bilateral lumbar sympathectomy under general anaesthesia. The cause of the death, as confirmed by hospital records, was malignant hyperthermia. No genetic counselling was given at that time; this omission has since been corrected.

Early diagnosis and the availability on the premises of the necessary drugs and equipment to treat such a complication probably saved this child"s life.

In 1975, "La Corporation Professionnelle des Médecins du Québec" published standards for practice of anaesthesia outside a hospital setting. The compliance of the dental clinic with such standards has contributed to the avoidance of a catastrophe.

\section{REFERENCES}

1. King, J.O., Denborough, M.A., \& Zapf, P.W. linheritance of malignant hyperthermia. Lancet Feb. 12.365 (1972).

2. Greenfield, S., Bronsther, B., Abrams, M.W.. \& Rosenberg, H. The malignant hyperthermia syndrome. Clinical Pediatrics 16:808 (1977).

3. Britt, B.A., Locher, W.G., \& Kalow, W. Hereditary aspects of malignant hyperthermia. Can. Anaes. Soc. J. 16: 89 (1969).

4. Moulds, R.F.W. \& Denborough, M.A. Biochemical basis of malignant hyperpyrexia. B.M.J. 2: 241 (1974).

5. Kaplan, A.. Bergeron, P.S., Gregg, S.A., \& CURLESS, R.G. Malignant hyperthermia associated with myopathy and normal muscle enzymes. J. of Ped. 91: 431 (1977).

6. Vaughan, V.C.. MCKay, R.J.. \& Nelson, W.E. Nelson Textbook of Pediatrics, Ed. 10, Saunders, p. 1547 (1975).

7. Ellis, F.R., Clarke, I.M.C., Modgill, M., Curie, S., \& Harriman, D.G.F. Evaluation of creatinine phosphokinase in screening patients for malignant hyperpyrexia. B.M.J. 3: 511 (1975).

8. BRITT, B. Personal communication. University of Toronto (Jan. 1978).

9. Rueller, J.B. Management of hyperthermia. Can. Med. Ass. J. 117: 1372 (1977).

10. Guide d'Exercice de l'Anesthésie, Corporation Professionnelle des Médecins du Québec, p. 3-4 \& suiv. (14 mars 1975). 